\title{
using parameterized calculus questions for learning and assessment
}

\author{
L. Descalço and Paula Carvalho \\ Mathematics Department \\ University of Aveiro \\ Aveiro, Portugal \\ luisd@ua.pt and paula.carvalho@ua.pt
}

\begin{abstract}
We have implemented a Web application reusing questions from two computer systems, true/false questions from PmatE (Projeto Matemática Ensino) and multiple choice questions from Megua (Mathematics Exercise Generator, University of Aveiro). Our application implements a Bayesian user model for diagnosing student knowledge in the topics covered. In this article we propose the use of this system for both learning and assessment in a calculus course, encouraging the students to work during the semester without increasing the work load for teachers.
\end{abstract}

Keywords - Assessement; bayesian user model; independent learning, paremeterized questions, e-learnnig .

\section{INTRODUCTION}

Nowadays there exists an enormous amount of information available on the internet and computer systems useful for learning. As a natural consequence, a student can easily get lost. We focus on a simple tool for helping to guide students on the basic competencies in several mathematics courses, including calculus with one variable, calculus with several variables, mathematics for secondary school and mathematics for basic school. We have a purposed set of topics, not specific to particular courses, but consensual enough for being used in several similar courses.

Questions from two parameterized question generation computer systems are used, true/false questions from PmatE (see $[3,17]$ ), and multiple choice questions from Megua (see $[6,12])$. We have a set of selected questions from PmatE (presently 65 parameterized question generator templates), that have been reclassified and associated with the relevant topics in our conceptual map. Also, many multiple choice questions from Megua (presently 3041 questions) are being used the same way with the same purpose.

The main element of our Web application, that we call "Independent Study", presents the course to the student and allows him/her to select a topic, what makes a question, having some relation to the select topic, to appear. The student can then answer the question and has immediate feedback, being informed if her/his answer is correct or not as well as, for the multiple choice questions, providing a detailed solution for the problem. The student has also information about his general progress in the course. Progress bars associated with the concepts increase if the student answers questions correctly and decrease if she/he fails. Several progress bars are in fact affected when an evidence is provided, what is computed using a bayesian network (see [9,10]) with all concept and evidence nodes (see [4]).

We are using this system in the current semester, in a course of calculus with several variables, both for learning and assessment. Our aim is to encourage the students to work during the semester without increasing the work load for teachers.

\section{STATE OF THE ART}

From 1989, PmatE has been developing and using parameterized questions for computer tests about mathematics. The basic idea is that, for example, instead of creating a database of questions like " $1+1=2$ ?" we can create a database of question templates like " $a+b=c$ ?" together the information that the parameters "a,b,c" will be randomly chosen by the computer, form set " $\{1,2,3\}$ ". Based on this basic idea, many learning objects, consisting of an initial text together with at least four statements about the text, have been developed by PmatE. The initial text and the statements include some parameters that can be numbers, parts of text, or even pictures. These question generator template (QGT) specify the sets from where the parameters are randomly instantiated by the computer in runtime. A question generated by one of these templates, consist of an initial text and four randomly selected statements related to that text, as illustrated in Figure 1. In this example, not only the centers of the ellipses are parameterized but also the corresponding pictures.

This technology has been used both for knowledge diagnosis and in the yearly science competitions in the University of Aveiro, with the participation of about 12000 students in the three days of the competition. 


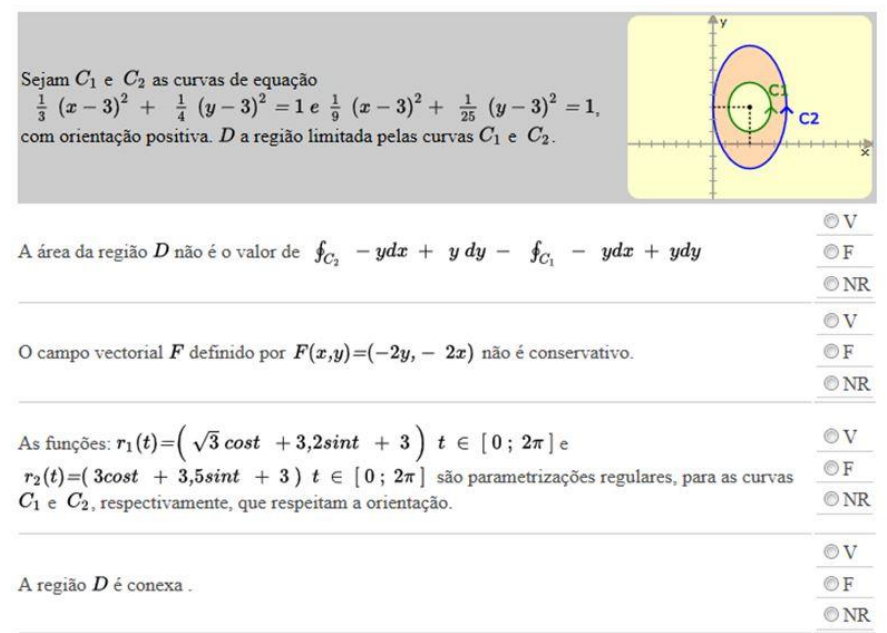

Figure 1: A question from PmatE

Project Megua, more recent, allows the creation of parameterized questions in PDF format for printing, in LaTeX for using in texts and exams, and also multiple choice questions for Web applications, as illustrated in Figure 2, the kind of questions we use in our application. A main advantage of this system is that the teacher can produce the question without the need of a programmer, as it was the case with PmatE. Nevertheless this requires the teacher to do some programing, although elementary. A new platform from PmatE for producing questions without the need of a programmer that also does not require the teacher to do any programming is currently being tested. Of course the functionality is limited compared with Megua where the teacher has access to a programing language (Sage Notebook; see [19]).

$$
\begin{aligned}
& \text { Seja } f \text { uma função integraivel. Considere o integral } \\
& \qquad \iint_{D} f(x, y) d x d y \\
& \text { onde } \\
& D=\left\{(x, y): \frac{13}{4} \leq x \leq \frac{17}{4}, \quad 5 x \leq y \leq 4 x^{2}\right\} .
\end{aligned}
$$

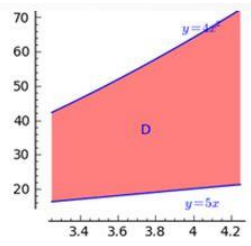

Qual das seguintes afirmaçęes è verdadeira?

$$
\begin{aligned}
& \iint_{D} f(x, y) d x d y=\int_{\frac{13}{4}}^{\frac{14}{4}} \int_{5 x}^{4 x^{2}} f(x, y) d y d x \\
& \iint_{D} f(x, y) d x d y=\int_{5 x}^{4 x^{2}} \int_{\frac{y}{4}}^{\frac{17}{4}} f(x, y) d x d y
\end{aligned}
$$

$$
\iint_{D} f(x, y) d x d y=\int_{\frac{y}{4}}^{\frac{y y}{4}} \int_{4 z^{2}}^{5 x} f(x, y) d y d x
$$

$$
\iint_{D} f(x, y) d x d y=\int_{\frac{y}{4}}^{\frac{1 y}{4}} \int_{\frac{10}{4}}^{\frac{20}{4}} f(x, y) d y d x
$$

Figure 2: A question from Megua
Other two projects in Portugal with question generation templates are Passarola, from University of Minho (see [2]), and Mito from Escola Superior de Tecnologia e Gestão de Leiria (see [16]).

Our main contribution is to reuse QGTs from our two systems and to make them available on the Web with a Bayesian user model for learning guidance. In this article we focus on a particular use of this system both for learning and assessment in calculus with several variables. For this propose we had to develop many parameterized questions in order to completely cover the topics in the course.

\section{THE WEB APPLICATION}

With the availability of Question Generation Templates from PmatE (presently 1252 for mathematics), it is natural to try to develop computer learning systems making use of them. We have already developed other Web applications using QGTs from PmatE (see [1]) and we have now implemented a Web application, named Siacua (which stands for Interactive System form Learning by Computer in Portuguese; available in [18]). This application implements a bayesian user model, with an algorithm for knowledge propagation. Each student has an associated bayesian network, where each node represents a topic, although we sometimes call it a concept node to distinguish from evidence nodes. Before the student starts answering questions, his/her bayesian network contains only concepts, it is the conceptual map of the subject being studied. Each time the student answers a question, a new node is added to the network, an evidence node, connected with the concepts it involves, and the student knowledge is propagated.

Siacua is an open system in the sense that students can see their progress, which is shown in the form of progress bars. The bayesian network associated with the student is used to update all progress bars each time she/he provides evidence by answering a question. The interaction is minimal, consisting of answering true/false questions form PmatE, multiple choice questions from Megua and selecting questions. The selection of questions can be made by clicking the corresponding progress bar, what randomly selects a question related with the corresponding concept, or by introducing the question number. These question numbers are only shown to students in the moment they are answering the question. This allows students to identify the questions for answering again or interacting with teachers and colleagues. Since a question may be associated with several topics, when a student clicks a topic, only questions not associated with topics appearing afterwards can be presented. So, if we follow the order of the index in our application, we are sure that it cannot pose questions including topics we have not introduced yet in the classes. Of course a student can chose any topic and, in the case he/she clicks the course name, any question may appear. 


\section{LEARNING AND ASSESSMENT}

Siacua provides a very simple way of presenting questions to the student, and giving some feedback. The student knows immediately if his answer is right or wrong, can see the detailed solution and has information about his general progress in the course, given by the progress bars, as illustrated in Figure 3.

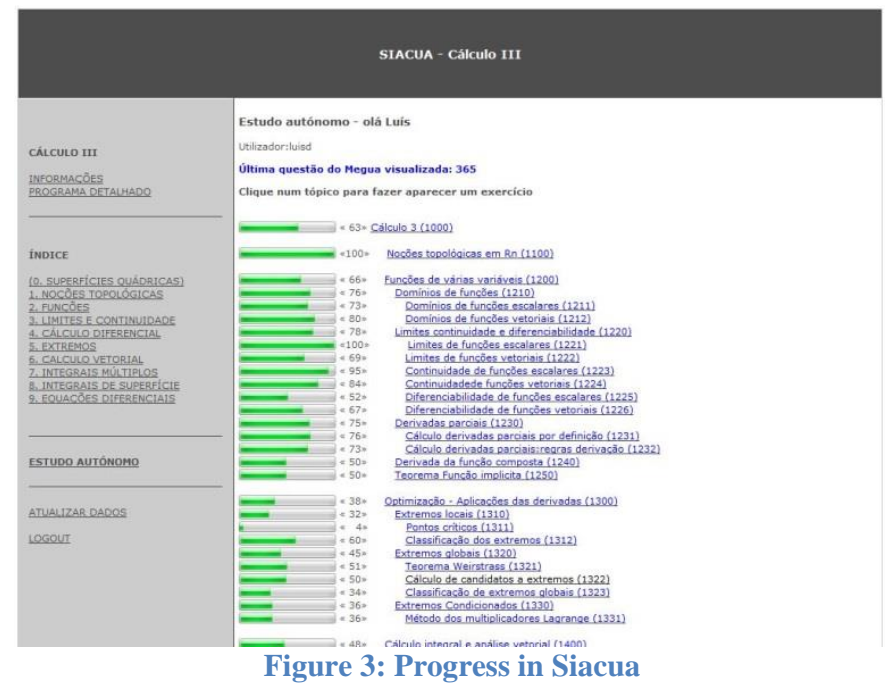

The application can be used in computers and mobile devices. Students use it as a tool for studying and teachers use it sometimes in the classes, as well. A teacher can see the progress of each student in the application and also the average progress of each class in each topic being studied.

We allow the students to see all questions and learn with the system, and then we simply set the dates where the classifications of some selected progress bars are collected from the application to be used for assessment. With this approach we intend to motivate the students to do some work along the semester instead of leaving the study to the days before the main assessment tests, what normally occurs with unsatisfactory results. Moreover, this approach does not increase the work load of teachers, since the Web application was already available for learning, and is simply being reused for assessment without any change.

Although the student can select a question in Siacua by choosing the number that identifies it, she/he can only answer questions that appear randomly when he/she clicks a progress bar. The idea is to allow the student to see all questions he/she wants while learning, but not allow him to choose the questions to answer for assessment. The number of available generated questions is high but of course the system can still be cheated. We also leave it clear that it is convenient for students to use it appropriately, since we will assume that in the main assessment tests, with much more weight in the final classification formula $(80 \%$ for written tests and $20 \%$ for
Siacua). We note that the student has the option of being assessed only by the written tests.

Collaboration of students and teachers is done in person and in a Facebook group where questions from Siacua are mentioned by their numbers.

\section{SYSTEM DIAGRAM}

Questions from PmatE are available, through a Web service, for use in Siacua. We have selected some of these many questions and classified them using our concept maps. Technically, the Web application sends a request to a Web service from PmatE, sending the question id, and the Web service generates the question using the corresponding QGT. We note that this is done in runtime. The parameters are randomly generated when the request is made.

Questions from Megua are previously generated. This means that for a given QGT, a set of instances is created and stored in Siacua database, before they can be used. This option has advantages and disadvantages. The main disadvantage is that the number of questions generated by a given Template is fixed. Nevertheless, this number can be high, with some cost on memory use in the database, and in practice the behavioral is the same as with runtime generated questions. An obvious advantage is speed, since question instances are already available without the need of computation. Another advantage is that the teacher can choose some question instances with better parameters, producing better looking and more interesting questions than those obtained allowing the computer to randomly choose any parameters.

A question can be created using Megua and sent to Siacua being immediately available online. A teacher can experiment inside Megua question creation environment and select several instances with convenient question parameters. Then he has to associate the question to concepts from the concept map of the course, and define the parameters for the bayesian network. Finally the teacher can send several instances of the questions to Siacua (Implemented using C\#, SQL, Mathjax and Smile; see [7, 8, 11]).

Figure 4 provides a simple diagram illustrating the interaction of the several elements in our system. Teachers can create or select existing questions in PmatE and Megua and these questions can be directly accessed from Siacua. Teachers have also several special operations in Siacua, like observing the students' progress, as we have already mentioned. Students interact with Siacua where they can see and answer questions.

\section{THE USER MODEL}

The conceptual map of the course is represented by a graph where the nodes represent "concepts" and edges represent 
aggregation relations ("is part of"). With this graph we build a Bayesian network, adding conditional probabilities to the nodes. These probabilities are computed from the specified weights of each child "concept" in the parent node. So, for example, if Derivatives has weight 0.5 , Integrals weight 0.3 and Differential equations has weight 0.2 in Course Calculus, then, the probability for a student to know Calculus, assuming knowledge of Derivatives and Integrals is 0.8 . We just add the weights.

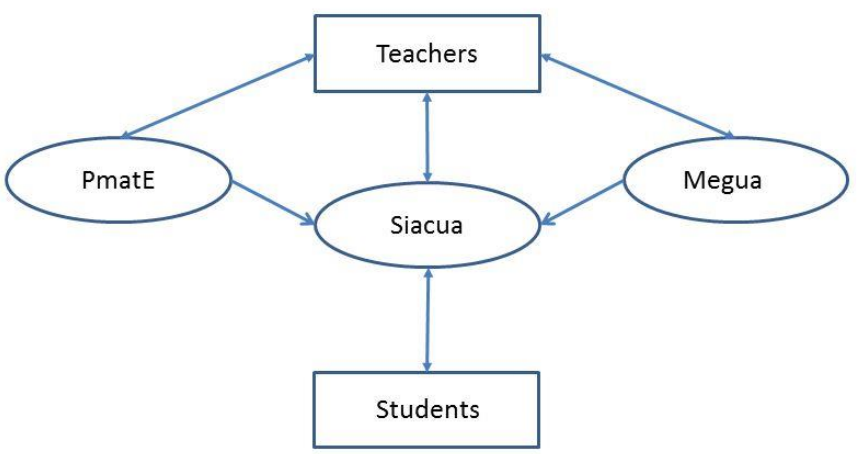

Figure 4: System diagram

When a student answers questions, evidence nodes are added to the Bayesian Network, representing evidences. The conditional probabilities associated with these evidence nodes are computed from a set of parameters: a list of concepts and the corresponding weights, parameters "slip" (probability of giving a wrong answer, knowing all concepts it involves), "guess" (probability of giving a correct answer without knowing any concept it involves), "level" (difficulty level) and "discr" (discrimination factor).

Hence, when a teacher creates a question in Megua, the parameters for the bayesian network have to be set and sent to Siacua, together with the question itself.

The user model defines a way to compute all conditional probabilities in the Network from the parameters. The probability of giving a correct answer to a question without knowing any concept in involves is the value of "guess" and the probability of giving a correct answer to a question knowing all concepts it involves is equal to 1-“slip". To compute the other probabilities, namely the probabilities of giving a correct answer knowing some concepts and not knowing others, we use a function ( $\mathrm{G}$ function used in IRT item response theory) defined by

$$
G(x)=1-\frac{(1-c)\left(1+e^{-1.7 a b}\right)}{1+e^{1.7 a(x-b)}}
$$

where $a$ is the value of "discr", $b$ the value of "level", $c$ is the value of "guess" and $s$ is the value of "slip", with graph illustrated by Figure 5 .

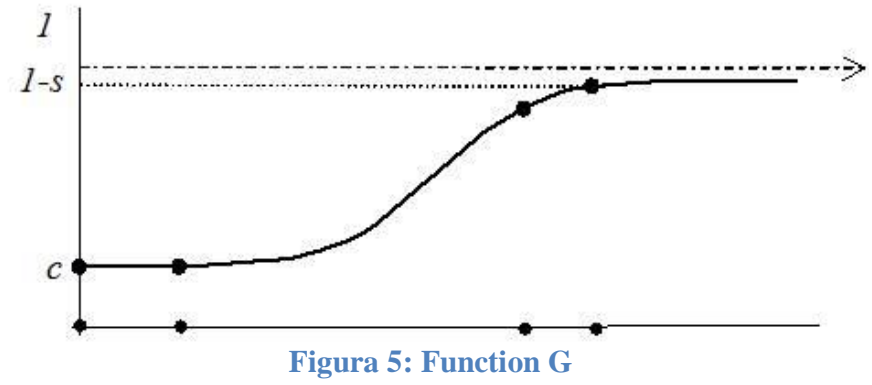

The user model we use has been proposed, described in detail and tested with simulated students in $[14,15]$ and has been tested with real students in [5].

\section{CONCLUSIONS AND FUTURE WORK}

Based on two inquiries from the last two years, on courses about calculus with several variables, it is clear that students value our work and prefer to have questions presented this way than simply made available on the University e-learning system (Moodle). The average answer to one of the questions in the two inquiries: "It is better to have a dedicated application, like Siacua, for autonomous study, than to have only study material available on e-learning (Moodle)" (in a scale 1-5 where 1 stands for "disagree completely" and 5 stands for "agree completely"), in a sample of 100 students, was 4,6 in the year $2013 / 2014$ and 4.5 , in a sample of 300 students, in the year 2014/2015 (first semester).

We are reusing a Web application designed for learning as an optional tool for assessment, what encourages the students to work along the semester without increasing the work load for teachers. Motivated by the very positive student feedback, both in the inquires and directly to teachers, we plan to continue the development of our parameterized questions based system, increase the number of available questions and analyze the data collected by the application and relate it with the final results in the course.

With many students making a regular use of Siacua and considering it an important complement, we believe it has a motivating effect in the teaching-learning process. Moreover, using Siacua in assessment, more students will use it for study and making a more careful use of it. Since this methodology will encourage students to work during the semester it will contribute to the success in the course of calculus with several variables, a goal not easy to achieve. We hope to confirm these statements in the end of the current semester, when the experience ends, by analyzing the data collected by Siacua and the results of students in assessment. 


\section{ACKNOWLEDGEMENTS}

This work was supported by CIDMA ("Center for Research \& Development in Mathematics and Applications"') and FCT ("FCT- Fundação para a Ciência e a Tecnologia") through project UID/MAT/04106/2013.

\section{REFERENCES}

[1] Maribel Aires; L. Descalço. "A computer aided learn by example system", proceedings of the conference ICMSE 2006, Internationa Conference in Mathematics, Sciences and Science Education, Universidade de Aveiro, 2006.

[2] José João Almeida, Isabel Araújo, Irene Brito, Nuno Carvalho, Gaspar J Machado, Rui MS Pereira, Georgi Smirnov, "PASSAROLA: HighOrder Exercise Generation System", CISTI 13, 2013.

[3] António Batel Anjo; Rui G. Isidro; Paula Oliveira; Sónia Pais; Joaquim Sousa Pinto. "TDmat - Mathematics Diagnosis Evaluation Test for Engineering Sciences Students", co-author ( 6 authors), International Journal of Mathematical Education in Science and Technology, v38 n3 p283-299, ISSN: 0020-739X (Print), 1464-5211 (Online), 2007.

[4] Paula Carvalho, J.P. Cruz, L. Descalço, Paula Oliveira and Dina Seabra. "Using bayesian networks and parameterized questions for independent study", submited.

[5] Gladys Castillo, Eva Millán, L. Descalço, Paula Oliveira, Sandra Diogo: Using bayesian networks to improve knowledge assessment. Computers \& Education 60(1): 436-447, 2013.

[6] Pedro Cruz, Paula Oliveira, Dina Seabra. "Exercise templates with Sage.”, Tbilisi Mathematical Journal, 5(2), 37-44, 2012.
[7] Marek J. Druzdzel. "SMILE: Structural Modeling, Inference, and Learning Engine and GeNIe: A development environment for graphical decision-theoretic models" (Intelligent Systems Demonstration). In Proceedings of the Sixteenth National Conference on Artificial Intelligence (AAAI-99), pages 902-903, AAAI Press/The MIT Press, Menlo Park, CA, 1999.

[8] Genie \& Smile, Decision Systems Laboratory of the University of Pittsburgh and available at http://genie.sis.pitt.edu

[9] Finn V. Jensen and Thomas D. Nielsen. Bayesian Networks and Decision Graphs. Information Science and Statistics. Springer, 2007.

[10] Timo Koski and John M. Noble. Bayesian Networks, An Introduction. Wiley Series in Probability and Statistics. Wiley, 2009

[11] Mathjax. Available at http://www.mathjax.org/about/.

[12] Mathematics Exercise Generator, Universidade de Aveiro (Megua), http://cms.ua.pt/megua/

[13] MEGUA package for parameterized exercises in Sage Mathematics. Available at http://cms.ua.pt/megua.

[14] Eva Millán: Thesis: Bayesian system for student modeling. AI Commun. 13(4): 277-278, 2000

[15] Eva Millán, José-Luis Pérez-de-la-Cruz: A Bayesian Diagnostic Algorithm for Student Modeling and its Evaluation. User Model. UserAdapt. Interact. 12(2-3): 281-330, 2002.

[16] Paiva, R. (2011). Conteúdos didáticos multimédia, testes e exercícios de treino de Matemática online. Atas do encontro da SPM Leiria 2010 Boletim especial da Sociedade Portuguesa de Matemática, pp. 119-123.

[17] PmatE (Projeto Matemática Ensino), http://pmate.ua.pt

[18] Siacua (Interactive Computer Learnig System, University of Aveiro). Available at See http://siacua.web.ua.pt

[19] William A. Stein et al. "Sage Mathematics Software (Version 4.6.1)", The Sage Development Team, http://www.sagemath.org 